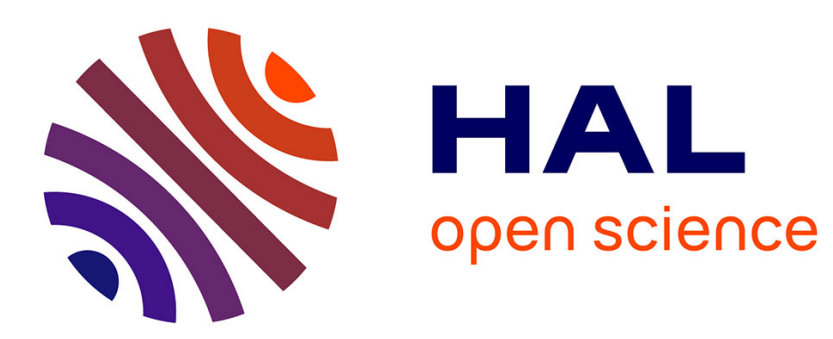

\title{
OECD-NEA Expert Group on Multi-Physics Experimental Data, Benchmarks and Validation
}

T. Valentine, M. Avramova, A. Petruzzi, J.-P. Hudelot, K. Ivanov, U. Rohatgi, K. Velkov

\section{- To cite this version:}

T. Valentine, M. Avramova, A. Petruzzi, J.-P. Hudelot, K. Ivanov, et al.. OECD-NEA Expert Group on Multi-Physics Experimental Data, Benchmarks and Validation. ICONE26 - 26th International Conference on Nuclear Engineering, Jul 2018, London, United Kingdom. cea-02339340

\section{HAL Id: cea-02339340 https://hal-cea.archives-ouvertes.fr/cea-02339340}

Submitted on 23 Mar 2020

HAL is a multi-disciplinary open access archive for the deposit and dissemination of scientific research documents, whether they are published or not. The documents may come from teaching and research institutions in France or abroad, or from public or private research centers.
L'archive ouverte pluridisciplinaire HAL, est destinée au dépôt et à la diffusion de documents scientifiques de niveau recherche, publiés ou non, émanant des établissements d'enseignement et de recherche français ou étrangers, des laboratoires publics ou privés. 


\author{
Proceedings of the $26^{\text {th }}$ International Conference on Nuclear Engineering \\ ICONE26 \\ July 22-26, 2018, London, England
}

ICONE26-81571

\title{
OECD-NEA EXPERT GROUP ON MULTI-PHYSICS EXPERIMENTAL DATA, BENCHMARKS AND VALIDATION
}

\author{
Timothy Valentine \\ Oak Ridge National Laboratory \\ Oak Ridge, TN, USA
}

\author{
Kostadin Ivanov \\ North Carolina State University \\ Raliegh, NC, USA
}

\author{
Maria Avramova \\ North Carolina State University \\ Raliegh, NC, USA
}

\author{
Alessandro Petruzzi \\ NINE - Nuclear and Industrial \\ Engineering \\ Lucca, Italy
}

\author{
Jean-Pascal Hudelot \\ CEA Cadarache \\ Saint Paul Lez Durance, France
}

Uprendra Rohatgi

Brookhaven National Laboratory

Upton, NY, USA

\author{
Kiril Velkov \\ Gesellschaft für Anlagen- und Reaktorsicherheit \\ Garching b. Munchen, Germany
}

\section{ABSTRACT}

High-fidelity, multi-physics modeling and simulation (M\&S) tools are being developed and utilized for a variety of applications in nuclear science and technology and show great promise in their abilities to reproduce observed phenomena for many applications. Even with the increasing fidelity and sophistication of coupled multi-physics M\&S tools, the underpinning models and data still need to be validated against experiments that may require a more complex array of validation data because of the great breadth of the time, energy and spatial domains of the physical phenomena that are being simulated. The expert group on Multi-Physics Experimental Data, Benchmarks and Validation (MPEBV) of the Nuclear Energy Agency (NEA) of the Organization for Economic Cooperation and Development (OECD) was formed to address the challenges with the validation of such tools. The work of the MPEBV expert group is shared among three task forces to fulfill its mandate and specific exercises are being developed to demonstrate validation principles for common industrial challenges. This paper describes the overall mission of the group, the specific objectives of the task forces, the linkages among the task forces, and the development of a validation exercise that focuses on a specific reactor challenge problem.

\section{INTRODUCTION}

High-fidelity, multi-physics modeling and simulation (M\&S) tools are being developed and utilized for a variety of applications in nuclear science and technology and show great promise in their abilities to reproduce observed phenomena for many applications. These M\&S tools enable rigorous modeling of coupled behaviors including among other things reactor physics, thermal hydraulics, fuel performance, structural mechanics, and materials chemistry. Even with the increasing fidelity and sophistication of coupled multi-physics M\&S tools, the underpinning models and data still need to be validated against experiments. This may require a more complex array of validation data because of the significant range of the time, energy and spatial domains of the physical phenomena that are

\footnotetext{
1 Notice: This manuscript has been authored by UT-Battelle, LLC, under contract DE-AC05-00OR22725 with the US Department of Energy (DOE) The US government retains and the publisher, by accepting the article for publication, acknowledges that the US government retains a nonexclusive, paid-up, irrevocable, worldwide license to publish or reproduce the published form of this manuscript, or allow others to do so, for US government purposes. DOE will provide public access to these results of federally sponsored research in accordance with the DOE Public Access Plan (http://energy.gov/downloads/doe-public-access-plan).
} 
being simulated and also to address the validation of the coupling approaches. Coupling of two or more single physics codes may accentuate the importance of some parameters due to feedback effects that are not modelled when boundary conditions are used to couple codes; these potential multiphysics phenomena manifest themselves from multi-physics experiments. The validation challenge is further complicated by the fact that legacy experimental data for single or coupled physical phenomena may not be adequate for validation of highfidelity M\&S tools, the fact that there are few experimental facilities available for conducting experiments, and the fact that in some instances instrumentation and experimental techniques may not exist to validate some models or approximates.

The expert group on Multi-Physics Experimental Data, Benchmarks and Validation (MPEBV) of the OECD-NEA was created in 2014 to address the specific challenges with the validation of high-fidelity, multi-physics $M \& S$ tools. The aims of the group are to provide the member countries of the OECDNEA with consensus guidelines and recommendations for validating multi-physics M\&S tools, to evaluate legacy and new experiments for validation, and to demonstrate validation principles for specific industry challenge problems. These efforts are supported by three separate task forces that will be briefly described. The MPEBV is also currently developing a validation exercise that focuses on the simulation of coupled multi-physics experiments for fuels performance to demonstrate validation approaches for both traditional and novel M\&S tools that will also be discussed in detail. [1]

\section{MOTIVATION AND ORGANIZAITON OF THE MPEBV}

The use of computational methods continues to expand to meet the demands of the research community, designers, developers, operators and regulators. High-fidelity multiphysics computational tools offer the promise of more sophisticated simulations that provide abilities to model complex, coupled physical phenomena with increased accuracy and enhanced predictive capabilities. However, for this promise to be realized, the models, coupling approaches, and validation processes need to be established and the limits of validation data need to be understood. Validation of multi-physics M\&S tools requires that the coupled $M \& S$ tools be validated for each physical phenomenon that is simulated as well as the coupling among the physical phenomena. Three task forces were established in the expert group to address these challenges. Task force one primarily focuses on experimental data qualification and development of benchmarks, and is closely linked with the activities of task force two that primarily focuses on the development of validation principles and guidance. There are strong interdependencies between the activities of these two groups as shown in Figure 1. Task force three is primarily focused on demonstrating examples of the validation principles and approaches.

\section{Task Force One}

Task Force One (TF1) activities are organized into seven tasks with the first two tasks being shared with Task Force Two (TF2). The first task of TF1 and TF2 was to define the scope of multi-physics applications that would be considered by the group and establish a consistent set of terminology to categorize the phenomena and simulation processes. The members of the MPEBV elected to differentiate traditional multi-physics M\&S tools that have limited coupling of multiple physical phenomena from novel tools that utilize more tightly coupled phenomena and/or explicit coupling. The second task of TF1 focuses on the current status and expected needs for validation of multiphysics M\&S tools whereas the third task focuses on identifying the major validation challenges and priorities. The objective of the fourth task is to establish recommendations and processes for the evaluation of existing experimental data including uncertainty quantification. The fifth task proposes to examine the needs, options, recommendations and mechanisms for performing specific validation experiments whereas task six aims to identify developments in instrumentation, experimental methods, and data treatment that would be needed for validating novel M\&S tools. The final task of TF1 is to implement the guidance to develop multi-physics benchmark evaluations from existing or new experiments that serve as validation experiments. [2]

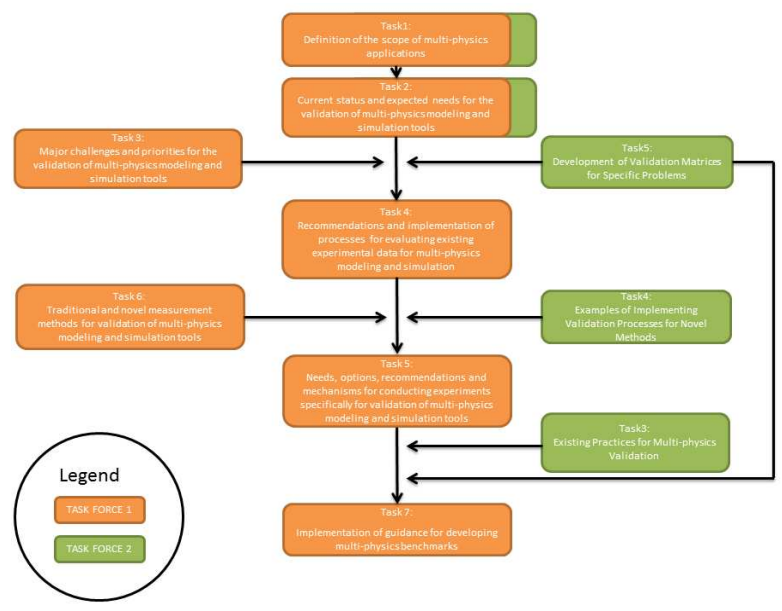

Figure 1. Organization and dependencies between activities of the two Task Forces [1].

\section{Task Force Two}

Task Force Two activities are organized into five tasks with the first two tasks being shared with TF1 that have been previously described. The third task of TF2 focuses on summarizing current approaches to multi-physics validation for traditional tools including approaches to sensitivity and uncertainty analysis. The objective of the fourth task is to establish recommendations for validation processes for novel 
multi-physics M\&S tools that also includes sensitivity and uncertainty analyses. The fifth and final task of TF2 is to develop validation matrices utilizing the phenomena importance ranking table (PIRT) process for specific challenge problems that were identified in task one of TF1 and TF2.

\section{Task Force Three}

Task Force Three (TF3) activities are organized into four tasks that involve the development of benchmark exercises that demonstrate validation processes for specific applications. The first involves the development of nuclear power plant benchmarks (VVER type reactor transients and PWR cycle depletion), the second involves the development of benchmarks exercises for startup experiments for transient reactors, and the third involves the development of benchmarks for research reactors. The fourth exercise is a validation exercise that intends to demonstrate the validation approaches for both traditional and novel multi-physics $M \& S$ tools for simulating ramp tests conducted in the Studsvik R2 reactor that will be further described.

\section{MULTI-PHYSICS PELLET CLADDING MECHANICAL INTERACTION VALIDATION (MPCMIV) EXERCISE}

While the capabilities of multi-physics M\&S tools can be demonstrated through the simulation of standard benchmark experiments, designers, operators and regulators must validate these codes for specific problems in order to quantify the limits of their applicability and the uncertainties in the predictive capabilities. The MPEBV expert group has selected several industry challenge problems as exercises for which to demonstrate validation principles and practices with the first focusing on approaches to validate both traditional and novel multi-physics $M \& S$ tools to simulate pellet cladding mechanical interaction experiments. Pellet cladding interaction (PCI) is of interest to both operators and regulators as PCI fuel failures can reduce reactor performance and limit the extent of power uprates, burnup, and fuel enrichments. With this exercise, the participants will be asked to simulate ramp tests performed in the Studsvik R2 Reactor [3]. The outcome of the simulations is less important than the principles, assumptions, and approaches that the participants implement in simulating the actual experiment. The participants will be asked to document these for both the single physics phenomena and the coupled physics phenomena. In addition, the participants will be asked to describe their approaches for conducting uncertainty analyses and to extrapolate these beyond the validation domain to the problem of interest when the validation data do not encompass the physics domain of the exercise.

The MPCMIV exercise is based upon experiments conducted at the Studsvik R2 reactor that requires coupling of reactor physics, thermal hydraulics, and fuel performance phenomena. For such an exercise, the coupling of the fuel behavior with reactor physics under irradiation is of primary importance while the coupling of thermal hydraulics being of less importance. The coupling of these phenomena is depicted in Figure 2 [4]. The fuel performance and reactor physics simulations are strongly coupled through the fuel temperature and reactor power for the ramp tests.
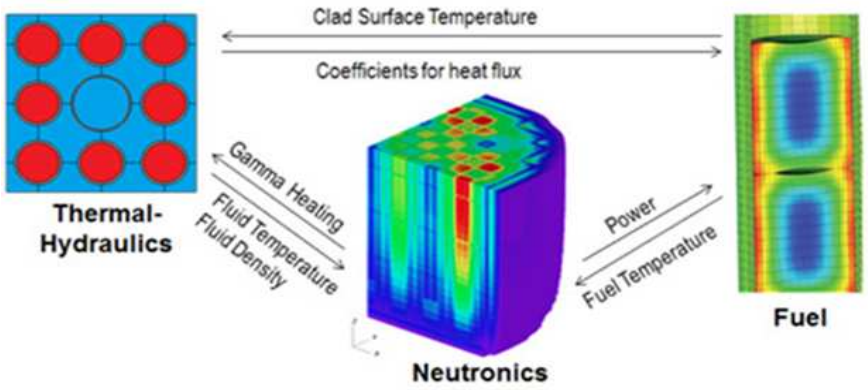

Figure 2. Coupling of reactor physics, thermal hydraulics and fuels performance simulations for the PCI problem [5].

The experiment that was selected for this exercise involved a cold ramp test conducted in the $\mathrm{R} 2$ reactor test loop that is positioned in the central region of the transient reactor core as shown in Figure 3 [5]. The aim of the experiment was to investigate the fuel response at cold criticality conditions to examine whether or not the potential fuel failure mechanisms might differ at temperatures below $100{ }^{\circ} \mathrm{C}$ than at normal operating conditions. The experiment was conducted in a stepwise fashion in that the rod was first held in cold conditions followed by a relatively fast transient in which the power generation in the rod increased from zero to $45 \mathrm{~kW} / \mathrm{m}$ in approximately 5 seconds. Within approximately 10 to 15 seconds the heat flux and fuel temperature reached their maximum upon termination of the power ramp. The experiment was subsequently shutdown manually.

The simulation of these experiments involves two distinct domains, i.e. the domain of the $\operatorname{rod}(\mathrm{R})$ and the domain of the reactor core $(\mathrm{C})$ since the fuel specimen is contained within a test loop that is held at conditions similar to those found in light water reactors. The in-core test loop is thermally insulated from the thermal response of the reactor core, and the fuel performance of the core fuel plates are of no consequence for this validation exercise. The primary coupling of these physical phenomena for this exercise are depicted in Figure 4. The participants will be asked to calculate multiple responses of interest (ROI) for the three physical phenomena and will be asked to provide these for multiple steps in the validation exercise. Some of the ROIs are from direct measurement while others are based on calculations conducted by Benchmark Organizers. 


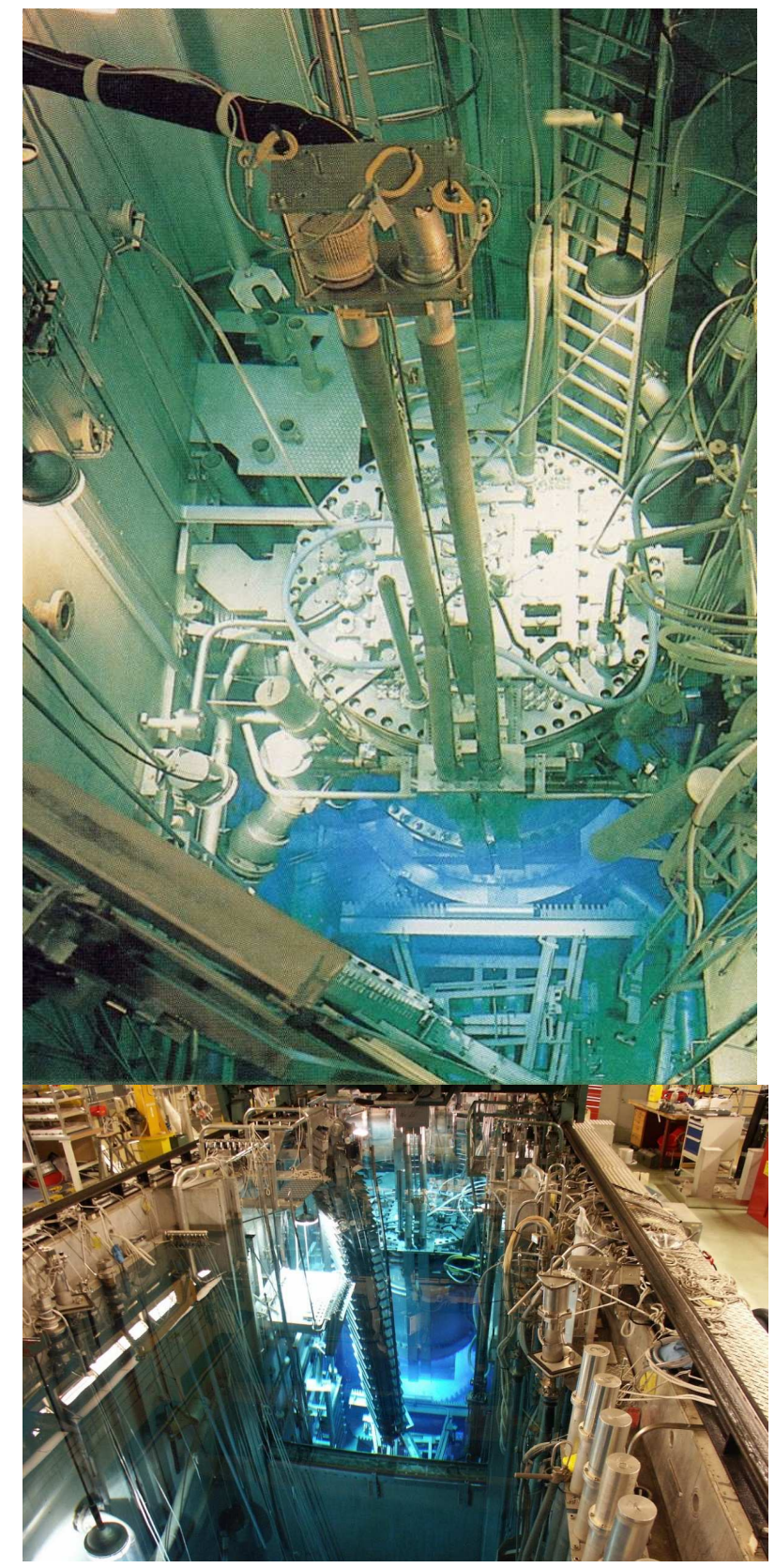

Figure 3. Photos of the Studsvik R2 reactor vessel [5].

The validation exercise is structured into three tiers in order to maximize participation by various groups. The first tier is targeted for novel M\&S tools that have the capability to model the 3D heterogeneous model for both the reactor core domain and the fuel rod domain; the second tier involves the use of a simplified model for novel M\&S tools that utilizes boundary conditions for the reactor physics models of the R2 reactor core; and the third tier involves the same simplified model of tier 2 but allows for the use of traditional M\&S tools.

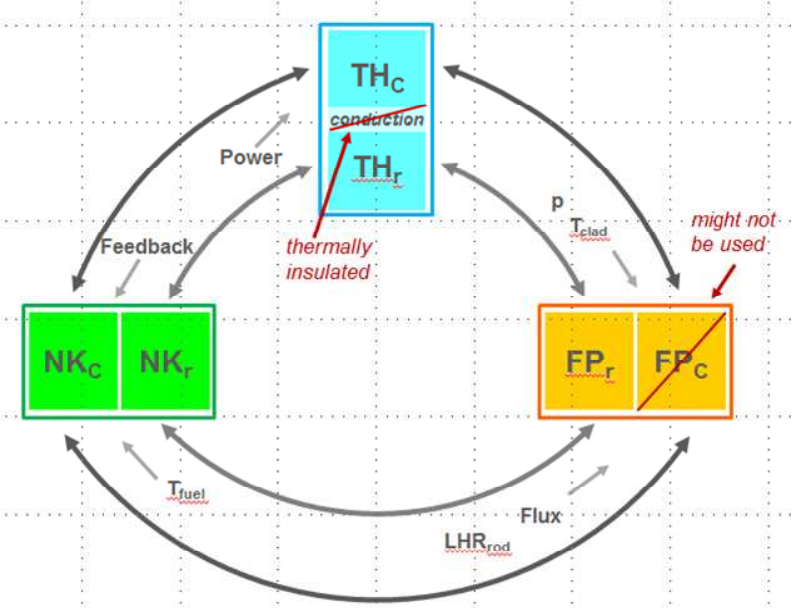

Figure 4. Coupling of phenomena for the MPCMIV validation exercise [6].

For each tier, the MPCMIV exercise will be structured into four phases: the development phase, the pre-qualification phase, the blind simulation, and the open phase. For each of these phases the participants will establish their validation requirements and assumptions that are made whether it be for steady state models or transient models. The development phase is focused on the modeling and includes the simulation of the steady state operation whereas the pre-qualification phase examines the transient response for a ramp test both with no rodlet (thermal-hydraulics and reactor physics involved) and with rodlet (all three physics phenomena involved) in the test loop. The blind simulation phase involves modeling of the BWR rodlet for a slightly different ramp test experiment (and with a different core configuration with respect to the one adopted for the pre-qualification phase) and it includes uncertainty analyses. The results from the blind test will not be attributed to any particular organization, but the participants will be encouraged to publish their results. The open phase is similar to the blind phase but will also include sensitivity analyses to provide quantification of the predictive capability of the $\mathrm{M} \& \mathrm{~S}$ tools.

Validation requirements will be established for each of the aforementioned steps. The participants will be required to quantify the accuracy of their simulations for each phase based on the approaches and data sets that were used to validate the $M \& S$ tools that were used. As an example, the development of the validation requirements of the reactor physics simulations for these phases is depicted in Figure 5. Such a process will be developed for each physical phenomenon as well as the coupled phenomena. 

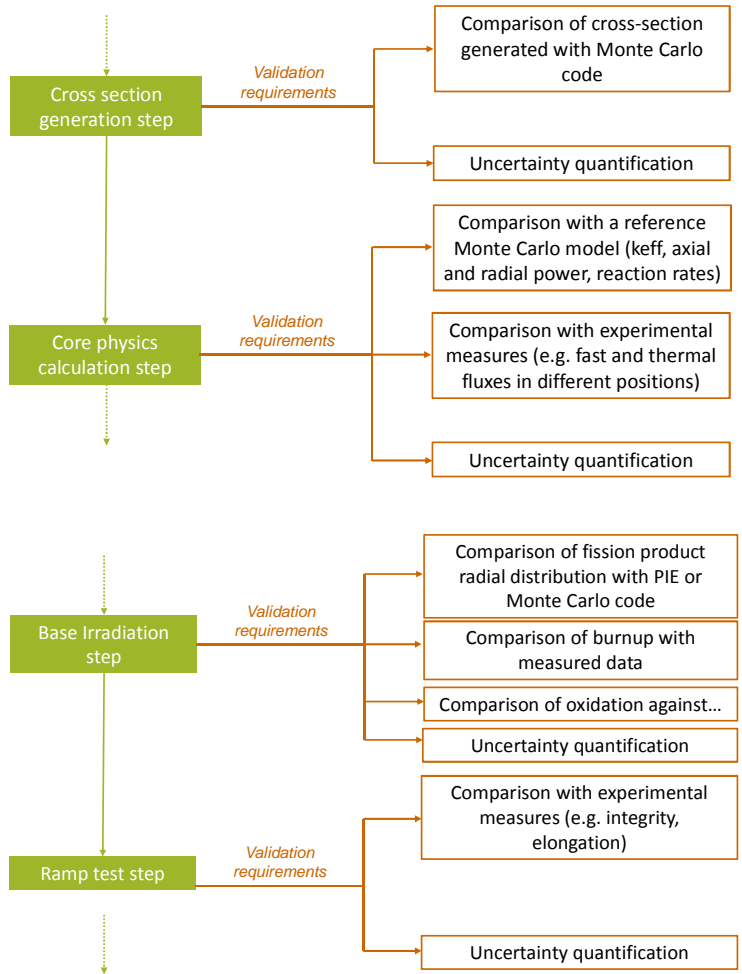

Figure 5. Reactor physics simulation phases for MPCMIV validation exercise [6].

\section{CONCLUSION}

The MPEBV expert group of the OECD-NEA was established to address challenges in validating multi-physics M\&S tools taking into account the lack of consensus on validation of coupled $M \& S$ tools, the availability of data to support such validation, and the impact of coupling mechanisms on validation. In addition to establishing validation principles and recommendations for evaluating the predictive capabilities of multi-physics M\&S tools, the group intends to demonstrate these approaches for a limited number of industry challenge problems. The first such example is the MPCMIV benchmark, and it will focus on simulating experiments that demonstrate the ability of both traditional and novel multi-physics simulation methods to replicate fuels performance measurements conducted in the Studsvik R2 reactor. While the outcomes of this exercise are of secondary importance, the exercise will provide the first opportunity to evaluate the validation principles and approaches and identify areas in which further development is needed including inadequacies in validation data sets, validation approaches, and methodologies to evaluate the predictive capability of the multi-physics M\&S tools. In addition, these exercises will contribute to the development of guidelines for validation of novel M\&S tools.

\section{REFERENCES}

1. P. Finck, T. Valentine, J. Dyrda, J. Gulliford, J.P. Hudelot, U. Rohatgi, "OECD-NEA Expert Group on Multi-Physics experimental Data, benchmarks and Validation", PHYSOR 2016 Conference, Sun Valley, ID, May 1-5, 2016.

2. K. Ivanov, T. Valentine, J-P. Hudelot, M. Avramova, U. Rohatgi, "Report on Task 1 of Task Force 1 and Task Force 2: Definition of the Scope of Multi-physics Applications," NEA/NSC/DOC (2016)1, OECD (2016).

3. M. Carlsson and U. Engman, "Test Facility and Experimental Technique for Fuel Ramp Tests," Report No. N(R)-99/063, Studvisk Nuclear, 15 Oct. 1999.

4. V. Mousseau, N. Dinh, "CASL Verification and Validation Plan”, CASL-U-2016-1116-000, June 30, 2016.

5. J. Karlson, "B64, Special Cold Ramp Test of Rod 2653," Report No. N-05/174, Studsvik Nuclear, 10 May 2007.

6. A. Petruzzi, "Motivations, Background, Structure and Status of the Multi-physics Pellet $\underline{\text { Clad }}$ Mechanical Interaction $\underline{\text { Validation (MPCMIV) }}$ Benchmark Specifications", 7th Meeting EGMPEBV, OECD Conference Centre, Paris, 19-21 September 2017. 